\title{
High-Quality Genome Sequence Resource of the Taro Pathogen Phytophthora colocasiae
}

\author{
Sachiko Masuda, ${ }^{1}$ Takashi Yaeno, ${ }^{2, \dagger}$ Hideaki Shibata, ${ }^{3}$ Shuuhei Yorozu, ${ }^{4}$ Satoki Yamamoto, ${ }^{4}$ \\ and Ken Shirasu ${ }^{1, \dagger}$ \\ ${ }^{1}$ RIKEN Center for Sustainable Resource Science, Yokohama, 230-0045, Japan \\ ${ }^{2}$ Department of Agriculture, Ehime University, Matsuyama, 790-8566, Japan \\ ${ }^{3}$ Ehime Research Institute of Agriculture, Forestry and Fisheries, Matsuyama, 799-2405, Japan \\ ${ }^{4}$ Agriculture and Horticulture Division, Agriculture, Forestry and Fisheries Department, Ehime Prefectural \\ Government, Matsuyama, 790-0001, Japan
}

Phytophthora colocasiae is an oomycete phytopathogen causing leaf blight and corm rot on taro (Colocasia esculenta), and its damage is a serious problem worldwide. Although the disease had not been reported in Japan for approximately 100 years, it began to spread in 2015.

Taro (C. esculenta) is a stable crop grown in the humid tropics of Asia, Africa, the Pacific, and the Caribbean (Kreike et al. 2004). In these regions, leaf blight and corm rot caused by $P$. colocasiae are the most limiting factor in taro production (Singh et al. 2012). P. colocasiae was first reported in Java by Raciborski (1900), moved from Java to Taiwan (Butler and Kulkarn 1913), and was thought to have spread to Japan and Hawaii in 1920 (Trujillo 1967). Even though Japan had been free of the epidemic for nearly 100 years afterward, severe damage caused by $P$. colocasiae was confirmed in 2015 , and the disease has continued to spread throughout the country, reducing yields by approximately $20 \%$ (Anonymous 2020). The cause of the current epidemic is not known. To understand what pathways caused the spread of the disease and whether the diversification of the pathogenicity is occurring, it is essential to collect genomic information on $P$. colocasiae in various regions. The genome of $P$. colocasiae 7290 from Vietnam was first sequenced by Vetukuri et al. (2018) using Illumina HiSeq2000. The short-read assemblies were fragmented and do not provide long-range haplotype and gene information. In this study, we report the genome of a new strain, EPC201509, isolated in Ehime Prefecture, Japan in 2015, sequenced using PacBio Sequel. The genomic DNA was extracted from mycelia cultured in liquid potato dextrose agar using the NucleoBond HMW DNA kit (Macherey-Nagel, Düren, Germany). The DNA concentration was measured using Nanodrop and Qubit (Thermo Fisher Scientific, Waltham, MA, U.S.A.).

The genomic DNA was sequenced by the long-read sequencer PacBio sequel. A DNA library was constructed with SMRTbell Express Template Prep Kit v 2.0 according to the manufacturer's protocol (Pacific Biosciences, Menlo Park, CA, U.S.A.) and was cut off at $20 \mathrm{kbp}$ using the Blue Pippin size selection system (Sage Science, Beverly, MA, U.S.A.). A total of $11.5 \mathrm{Gbp}$ was generated on a cell and assembled using SMRTLink v 8.0 (Pacific Biosciences) with minimum mapped length 1,000 bp. Finally, 428 contigs were generated with $143 \mathrm{Mbp}$ of the estimated genome size and $51 \mathrm{kbp}$ of $\mathrm{N}_{50}$ contig length (Table 1). The possibility that strain EPC201509, like strain 7290, is triploid has not yet been investigated

${ }^{\dagger}$ Corresponding authors: T. Yaeno; yaeno@agr.ehime-u.ac.jp, and K. Shirasu; ken.shirasu@ riken.jp The author(s) declare no conflict of interest.

Accepted for publication 2 December 2021.

\author{
Funding \\ Support was provided by the Japan \\ Society for the Promotion of Science \\ KAKENHI grant numbers \\ JP2OH05592 (to S. Masuda), \\ JP19K06056 (to T. Yaeno), and \\ JP17H06172 (to K. Shirasu).
}

\section{Keywords}

effector, oomycete, oomycete-plant interactions, PacBio Sequel, Phytophthora colocasiae, Phytophthora diseases, RXLR, taro, whole-genome sequence 
Table 1. Assembly results of EPC201509

\begin{tabular}{ll} 
Parameters $^{\text {a }}$ & Numbers \\
\hline Total length (bp) & $143,214,017$ \\
Number of the total contigs & 428 \\
$\mathrm{~N}_{50}$ contig (bp) & 509,258 \\
Largest contig (bp) & $2,045,440$ \\
GC contents (\%) & 50.9 \\
BUSCOs (eukaryota_odb10) & $94.9 \%$, with 484 complete, 2 fragmented, and 11 missing \\
BUSCOs (stramenopiles_odb10) & $100 \%$, with 100 complete \\
Total interspersed repeats (bp) & $71,765,842$ \\
Intact LTR-RTs (bp) & $17,399,166$ \\
Number of intact LTR-RT elements & $2,146(1,508$ is gypsy and 463 copia-like elements) \\
Number of predicted genes & 29,886 \\
RXLR genes & 289 \\
Crinkler genes & 416 \\
NPP1 genes & 105 \\
Elicitin genes & 74
\end{tabular}

${ }^{a}$ BUSCO = benchmarking universal single-copy orthologs and LTR-RTs = long-terminal repeat retrotransposons.

(Shrestha et al. 2017), and highly accurate long-read sequencing analysis would be required to determine whether this may have influenced the assembly. The draft genome of strain EPC201509 was evaluated by benchmarking universal single-copy orthologs (BUSCO v4) (Seppey et al. 2019) and showed 100\% completeness using stramenopiles_odb10. Because of the diversity in repetitive DNA content among Phytophthora spp. (Judelson 2012), the repeated sequences were searched using RepeatModeler v 2.0.1 (Flynn et al. 2020) and long-terminal repeat (LTR) retriever v2.9.0 (Ou and Jiang 2018). Finally, we detected $72 \mathrm{Mbp}$ of total interspersed repeats and $17 \mathrm{Mbp}$ of intact LTR retrotransposons (LTR-RTs) on the genome. The gene prediction was done using AUGUSTUS with the coding sequence datasets of $P$. capsici strain LT1534-B (GCA_016618375.1_Pcap_4.1_cds_from_genomic.fna), which belongs to the same clade 2 as $P$. colocasiae (Yang et al. 2017), as a training model (Hoff and Stanke 2013), resulting in 29,886 predicted genes with untranslated regions. The prediction of proteins involved in the pathogenicity using HMMER v3.3.2 with the corresponding profile HMMs resulted in 289 RXLR, 416 Crinkler, 105 NPP1, and 74 elicitin candidates (Table 1). Recently, the genome sequencing of a Chinese strain of $P$. colocasiae was reported using Oxford Nanopore Technologies PromethION (Wang et al. 2021).

This high-quality genome sequence resource will provide fundamental information for investigations of geographic origins, transmission routes, and virulence diversity. Further sequencing and comparative analysis of the genomes of strains from a variety of different regions will shed light on geographic origins, transmission routes, and virulence diversity. This Whole-Genome Shotgun project has been deposited at GenBank under the accession JAHCQX000000000. The version described in this article is version JAHCQX010000000.

\section{Acknowledgments}

We thank Y. Kanno for valuable information on how to culture the isolates for genomic DNA extraction.

\section{Literature Cited}

Anonymous. 2020. The Statistical Yearbook of MAFF. Statistics Department of the Ministry of Agriculture, Forestry and Fisheries, Japan.

Butler, E. J., and Kulkarn, G. S. 1913. Colocasia blight caused by Phytophthora colocasiae. Mem. Dep. Agric. India Bot. Ser. 5:233-259.

Flynn, J. M., Hubley, R., Goubert, C., Rosen, J., Clark, A. G., Feschotte, C., and Smit, A. F. 2020. RepeatModeler2 for automated genomic discovery of transposable element families. Proc. Natl. Acad. Sci. U.S.A. 117:9451-9457.

Hoff, K. J., and Stanke, M. 2013. WebAUGUSTUS-A web service for training AUGUSTUS and predicting genes in eukaryotes. Nucleic Acids Res. 41: W123-W128.
Judelson, H. S. 2012. Dynamics and innovations within oomycete genomes: Insights into biology, pathology, and evolution. Eukaryot. Cell 11:13041312.

Kreike, C. M., Van Eck, H. J., and Lebot, V. 2004. Genetic diversity of taro, Colocasia esculenta (L.) Schott, in Southeast Asia and the Pacific. Theor. Appl. Genet. 109:761-768.

Ou, S., and Jiang, N. 2018. LTR_retriever: A highly accurate and sensitive program for identification of long terminal repeat retrotransposons. Plant Physiol. 176:1410-1422.

Raciborski, M. 1900. Parasitic algae and fungi, Java. Batavia Bull. N. Y. State Mus. 19:189. 
Seppey, M., Manni, M., and Zdobnov, E. M. 2019. BUSCO: Assessing genome assembly and annotation completeness. Pages 227-245 in: Gene Prediction. Methods in Molecular Biology, Vol. 1962. M. Kollmar, ed. Humana, New York, NY, U.S.A.

Shrestha, S. K., Miyasaka, S. C., Shintaku, M., Kelly, H., and Lamour, K. 2017. Phytophthora colocasiae from Vietnam, China, Hawaii and Nepal: Intra- and inter-genomic variations in ploidy and a long-lived, diploid Hawaiian lineage. Mycol. Prog. 16:893-904.

Singh, D., Jackson, G., Hunter, D., Fullerton, R., Lebot, V., Taylor, M., Iosefa, T., Okpul, T., and Tyson, J. 2012. Taro leaf blight-A threat to food security. Agriculture 2:182-203.
Trujillo, E. E. 1967. Diseases of the genus Colocasia in the Pacific Area and their control. Pages 136-140 in: Proc. Second Int. Symp. Tropical Root and Tuber Crops, Honolulu, HI, U.S.A. University of Hawaii, Honolulu, HI, U.S.A.

Vetukuri, R. R., Kushwaha, S. K., Sen, D., Whisson, S. C., Lamour, K. H., and Grenville-Briggs, L. J. 2018. Genome sequence resource for the oomycete taro pathogen Phytophthora colocasiae. Mol. Plant-Microbe Interact. 31:903-905.

Wang, Z., Bao, J., Lv, L., Lin, L., Li, Z., Shi, M., Huang, Y., Wang, R., Li, B., Liu, P., and Chen, Q. 2021. Genome sequence resource of Phytophthora colocasiae from China using nanopore sequencing technology. Plant Dis. 105:4141-4145.

Yang, X., Tyler, B. M., and Hong, C. 2017. An expanded phylogeny for the genus Phytophthora. IMA Fungus 8:355-384. 